\title{
Characterizing the Response of Galloping Energy Harvesters Using Actual Wind Statistics
}

\author{
Mohammed F. Daqaq \\ Nonlinear Vibrations and Energy Harvesting Laboratory (NOVEHL) \\ College of Engineering and Science \\ Clemson University, Clemson, SC 29634 \\ email: mdaqaq@Clemson.edu
}

\begin{abstract}
In this paper, we incorporate actual wind statistics into the response of galloping energy harvesters and shed light onto the influence of the wind probability distribution on the average power as compared to the deterministic scenario. Specifically, we obtain an expression for the average output power of the harvester as function of the wind statistical averages, which are, in turn, obtained by fitting wind data using a Weibull Probability Density Function (PDF). The resulting expression is then used to demonstrate that knowledge of the actual PDF is essential for correct power predictions as well as for accurate electric load optimization. We discuss the influence of the wind direction on the average output power and show that the direction of the prevailing wind is not necessarily the ideal direction to maximize the average power.
\end{abstract}

\section{Introduction}

Flow energy harvesting has flourished in recent years with the basic premise of providing small levels of energy to power remote sensing units that are, otherwise, hard to access and maintain. Various fluidstructural interaction techniques were proposed to transduce a portion of the flow's kinetic energy into mechanical motion, which in turn, is transformed into electrical energy via an electromechanical coupling mechanism, e.g., piezoelectric or electromagnetic. Such techniques include, but, are not limited to, vortexinduced vibrations [1, 2], flutter [3-5] and the galloping instability [6-11].

As shown in Fig. 1, galloping can occur when incoming flow over a prism separates forming inner circulation under two nearly symmetric shear layers. Any small perturbations break the symmetry producing a negative surface pressure which generates a net aerodynamic lift on the body. As the wind speed exceeds the onset speed of galloping, the resulting aerodynamic lift overcomes the intrinsic dissipation forces causing the

(c) 2015. This manuscript version is made available under the Elsevier user license http://www.elsevier.com/open-access/userlicense/1.0/ 
oscillator to vibrate in the transversal direction. The motion of the prism increases the effective angle of attack which further increases the aerodynamic loading. The increased loading serves to amplify the oscillations' magnitude which results in self-sustained vibrations that build up until the amplitude is limited by nonlinearities in the aerodynamic and restoring forces. Unlike traditional rotary-type generators, galloping energy harvesters are very simple to design and fabricate, and hence, can be easily operated in various sizes and at different scales.

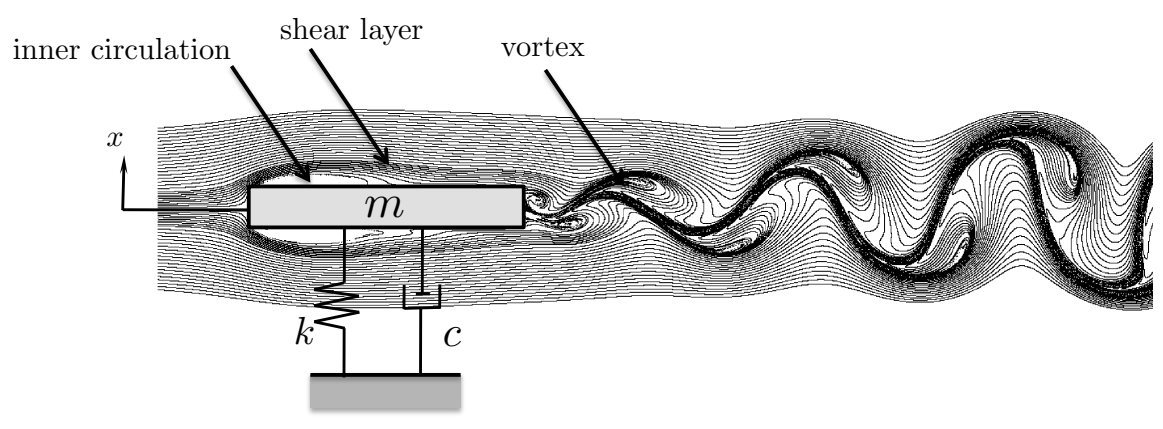

Figure 1: A schematic showing the flow streamlines past an oscillator.

The performance of a galloping energy harvester is usually assessed in a wind tunnel where the prism is mounted facing the flow direction and the wind speed is quasi-statically increased. At each step, the steadystate deflection, output voltage, and power are recorded. A diagram similar to the one shown in Fig. 2 is then generated illustrating the cut-in wind speed of the device as well as the amplitude of the steady-state output power at each wind speed. The process is then repeated for different electric loads to identify the optimal loading condition which enhances energy flow from the mechanical to the electrical subsystems.

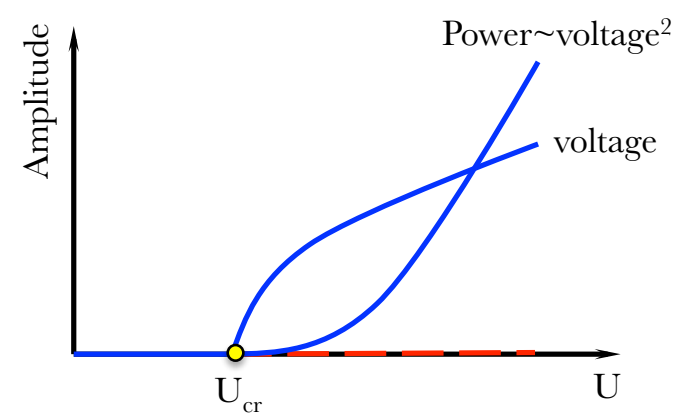

Figure 2: Bifurcation diagram of the steady-state response amplitude versus wind speed, $U$.

While this process is an essential first step in characterizing the harvester's response, it does not provide sufficient insight into the device's actual performance in a realistic setting where the wind speed is not constant but follows a specific annual probability density function (PDF). In fact, designing a galloping energy harvester based only on the knowledge of the average wind speed can yield inaccurate predictions regarding its average output power. For instance, consider a galloping energy harvester with a cut-in wind 
speed, $U_{c r}$. If the performance of the device was to be based solely on the average wind speed, then a location with an average wind speed below $U_{c r}$ will be rendered inadequate for the device's operation. However, with the knowledge that the wind statistics follow a PDF around the average wind speed, the device might actually generate sufficient power levels to maintain various sensing systems in the given location.

In this paper, we incorporate actual wind statistics into the response of galloping energy harvesters and shed light onto the influence of the wind statistics on the average power as compared to the deterministic scenario. We model the wind speed statistics using a general Weibull PDF and use it to obtain an expression for the average output power as function of the wind statistical averages. The resulting expression is used to study the effect of the PDF's shape and scale parameters on the average output power and optimal electric load. To achieve this goal the rest of the paper is organized as follows: Section 2 presents a general model of a galloping energy harvester assuming quasi-steady aerodynamics. Section 3 provides an expression for the average power as function of the wind statistical averages. Section 4 discusses the optimization of the electric load in the presence of probabilistic wind data. Section 5 presents a case study using data collected from different reporting stations in the United Kingdom (UK). Section 6 discusses the influence of the wind direction on the harvester's output power. Finally, Section 7 presents the important conclusions.

\section{Mathematical Modeling}

We consider the piezoelectric galloping energy harvester shown in Fig. 3 which consists of an oscillating spring-mass-damper system coupled to a resistive load, $R_{l}$ via a piezoelectric element of capacitance, $C_{p}$ and electromechanical coupling, $\theta$. The harvester is subjected to an aerodynamic lift force, $F_{y}$. The dynamics of the coupled system can be written as $[11,12]$

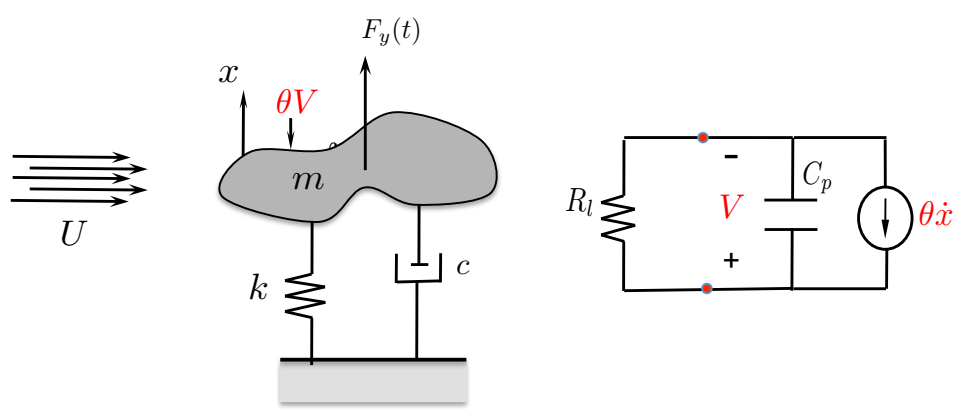

Figure 3: A lumped-parameters model of a galloping energy harvester.

$$
\begin{array}{r}
m \ddot{x}+c \dot{x}+k x-\theta V=F_{y}, \\
\theta \dot{x}+C_{p} \dot{V}+\frac{V}{R_{l}}=0,
\end{array}
$$


where the overdot represents a derivative with respect to time, $\tau, x$ is the displacement of the mass, $m$; $c$ and $k$ represent, respectively, the effective damping, and stiffness of the oscillator, and $V$ is the output voltage. In addition to the typical inertia, damping, and stiffness terms, Equation (1a) contains the electric damping force resulting from the backward coupling of the piezoelectric element.

The lift force $F_{y}$ is modeled using a quasi-steady assumption where the motion of the bluff body is assumed to be too slow compared to the motion of the fluid such that that the lift coefficient stays constant for a given angle of attack [12]. This guarantees that any disturbance experienced by the oscillating body is swept downstream sufficiently far by the time the body comes back to the same point for the disturbance to no longer affect the flow around the body. Under such conditions, the lift force can be expressed in terms of the oscillator's velocity and the wind speed as

$$
F_{y}=\frac{1}{2} \rho U^{2} L D\left[a_{1} \frac{\dot{x}}{U}-a_{3}\left(\frac{\dot{x}}{U}\right)^{3}\right],
$$

where $\rho$ is the air density, $U$ is the wind speed, $L$ and $D$ represent, respectively, the height and the cross-flow dimension of the prism, $a_{1}$ and $a_{3}$ are aerodynamic coefficients that depend on the geometry and aspect ratio of the bluff body. They are usually obtained empirically from normal aerodynamic force measurements on a static bluff body at different angles of attack [13].

From a physical perspective, the first term in Equation (2) is a negative linear damping term representing an energy pumping mechanism. This term captures the transfer of energy from the flow to the structure. Due to the negative damping, the response amplitude grows for small values of $\frac{\dot{x}}{U}$, up to the point where $\frac{\dot{x}}{U}$ is sufficiently large. At this point, the second term in Equation (2), which represents a nonlinear dissipation mechanism, becomes dominant. The nonlinear term serves to limit the growth of the amplitude which results in fixed-amplitude limit-cycle oscillations at a given wind speed.

Next, we nondimensionalize Equation (1) by introducing the following dimensionless terms:

$$
\begin{aligned}
& t=\omega_{n} \tau, \quad y=\frac{x}{D}, \quad v=\frac{C_{p}}{\theta D} V, \quad \mu=\frac{\rho L D^{2}}{4 m} \\
& \bar{U}=\frac{U}{\omega_{n} D}, \quad \kappa=\frac{\theta^{2}}{k C_{p}}, \quad \alpha=\frac{1}{R_{l} C_{p} \omega_{n}}, \quad \zeta_{m}=\frac{c}{2 m \omega_{n}}
\end{aligned}
$$

where $\omega_{n}=\sqrt{k / m}$ is the natural frequency of the mechanical oscillator, $t$ is the non-dimensional time, $v$ is the non-dimensional voltage, $\mu$ is the ratio between the flow inertia and that of the oscillator, $\bar{U}$ is the reduced wind speed, $\kappa$ is a non-dimensional electromechanical coupling coefficient, $\alpha$ is the ratio between the period of the mechanical system and the time constant of the harvesting circuit, and finally $\zeta_{m}$ is the mechanical damping ratio. Upon substituting the non-dimensional constants into Equation (1), we obtain the following non-dimensional equations:

$$
\begin{array}{r}
\ddot{y}+2\left(\zeta_{m}-\mu a_{1} \bar{U}\right) \dot{y}+y+\frac{2 \mu a_{3}}{\bar{U}} \dot{y}^{3}-\kappa v=0, \\
\dot{v}+\alpha v+\dot{y}=0 .
\end{array}
$$


Inspection of Equation (3) provides some additional insight into the dynamics of the coupled system. Near open circuit the time constant ratio, $\alpha$ approaches zero because the load resistance $R_{l}$ approaches infinity. In this case, the non-dimensional voltage, $v$, can be approximated by the non-dimensional response amplitude, $y$; and the two governing equations can now be reduced into a single equation in terms of $y$ as follows

$$
\ddot{y}+2\left(\zeta_{m}-\mu a_{1} \bar{U}\right) \dot{y}+(1-\kappa) y+\frac{2 \mu a_{3}}{\bar{U}} \dot{y}^{3}=0,
$$

Note that the effect of circuit dynamics is captured merely by a change in the stiffness of the oscillator. Furthermore, it can be clearly seen that the effective linear damping becomes negative when the reduced wind speed, $\bar{U}>\zeta_{m} /\left(\mu a_{1}\right)$. At this point, a dynamic instability (Hopf bifurcation) occurs as a result of two complex conjugate eigenvalues of the system's Jacobian matrix transversally crossing the imaginary axis. As a result, the response amplitude starts to grow up to the point where the positive nonlinear damping term becomes dominant, thereby limiting the response growth to a certain amplitude. Similar conclusions can also be deduced for the short conditions, with the main difference that the voltage approaches zero in this case.

An approximate analytical expression for the steady-state response of Equation (3) can be obtained using the method of multiple scales, see appendix A1 for details. The steady-state magnitude of the deflection and voltage are given by

$$
\begin{aligned}
& y=\frac{2}{\sqrt{3}} \frac{\left(\zeta_{m}+\zeta_{e}\right)}{\mu} \sqrt{\frac{\mu}{\left(\zeta_{e}+\zeta_{m}\right) a_{3}}\left(\frac{a_{1} \mu}{\left(\zeta_{e}+\zeta_{m}\right)} \bar{U}-1\right) \bar{U}}, \\
& v=\frac{2}{\sqrt{3}} \frac{\left(\zeta_{m}+\zeta_{e}\right)}{\mu \sqrt{1+\alpha^{2}}} \sqrt{\frac{\mu}{\left(\zeta_{e}+\zeta_{m}\right) a_{3}}\left(\frac{a_{1} \mu}{\left(\zeta_{e}+\zeta_{m}\right)} \bar{U}-1\right) \bar{U}},
\end{aligned}
$$

where $\zeta_{e}=\alpha \kappa /\left(2\left(1+\alpha^{2}\right)\right)$ is the electric damping resulting from energy dissipation in the load, $R_{l}$. It is worth noting that the voltage output of the harvester is only a scaled version of the response amplitude. They are related through the load resistance and the piezoelectric capacitance which are embedded within the dimensionless time constant ratio, $\alpha$. Note that the harvester produces voltage only when $\frac{a_{1} \mu}{\zeta_{e}+\zeta_{m}} \bar{U}>1$ and that the voltage increases with $a_{1}$. Furthermore, the output power is inversely proportional to the nonlinear aerodynamic coefficient, $a_{3}$, which, as mentioned earlier, serves to limit the growth of the response amplitude.

A dimensionless expression for the output power can be obtained by squaring Equation (6) and dividing by the load resistance. This yields

$$
\frac{P}{P_{0}}=\frac{4}{3} \frac{\mu}{a_{3}\left(\zeta_{e}+\zeta_{m}\right)} \bar{U}\left(\frac{a_{1} \mu}{\zeta_{e}+\zeta_{m}} \bar{U}-1\right),
$$

where $P_{0}=\theta^{2} D^{2}\left(\zeta_{e}+\zeta_{m}\right)^{2} /\left(C_{p}^{2} R_{l} \mu^{2}\left(1+\alpha^{2}\right)\right)$. 


\section{Average Power}

To determine the average power of the harvester, we average Equation (7) and obtain

$$
\frac{\langle P\rangle}{P_{0}}=\frac{4}{3} \frac{\mu}{a_{3}\left(\zeta_{e}+\zeta_{m}\right)}\left(\frac{a_{1} \mu}{\zeta_{e}+\zeta_{m}}\left\langle\bar{U}^{2}\right\rangle-\langle\bar{U}\rangle\right)
$$

where $\langle\square\rangle$ represents the statistical average, $\left\langle\bar{U}^{n}\right\rangle=\int_{0}^{\infty} \bar{U}^{n} H(\bar{U}) d \bar{U}$, and $H(\bar{U})$ is the PDF of the wind speed. Generally, based on experimental measurements, the PDF follows a Weibull distribution of the form $[14]$

$$
H(\bar{U})=\frac{\delta}{\beta}\left(\frac{\bar{U}}{\beta}\right)^{\delta-1} \exp ^{-\left(\frac{\bar{U}}{\beta}\right)^{\delta}}
$$

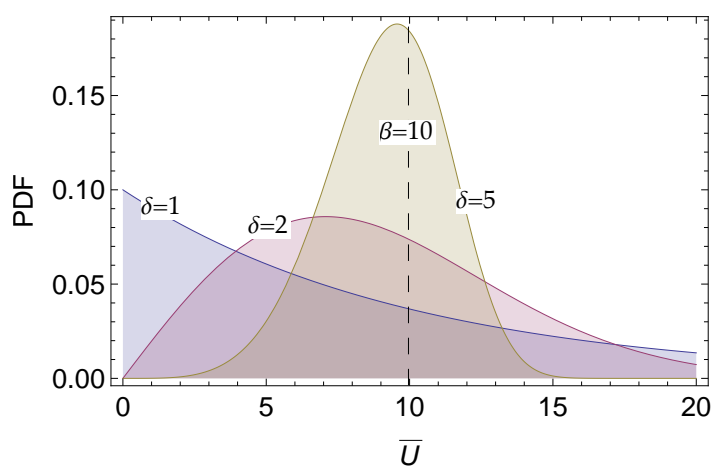

(a)

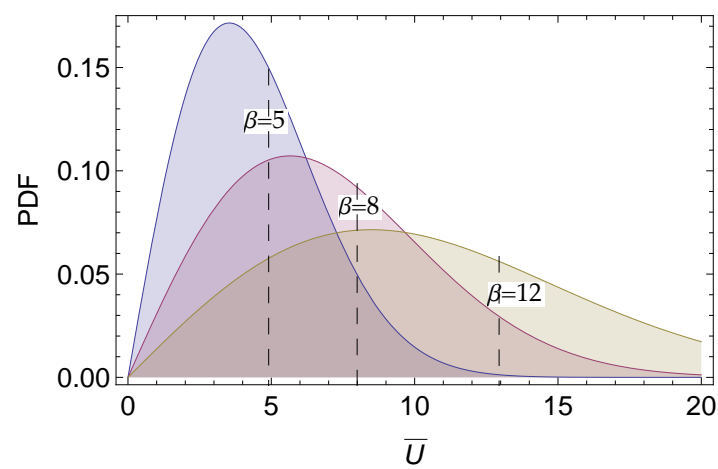

(b)

Figure 4: Weibull probability density function of the wind speed for (a) different shape parameters, $\delta$, and a scale parameter $\beta=10$, and (b) different scale parameters, $\beta$, and a shape parameter $\delta=2$.

where $\delta$ and $\beta$ are known, respectively, as the shape and scale parameters. As shown in Fig. 4 (a), $\delta$ controls the shape of the distribution. When $\delta \leq 1$, the PDF is exponentially correlated with lower wind speeds dominating the statistics. As $\delta$ increases, the peak probability shifts towards higher wind speeds but the PDF is still skewed towards smaller wind speeds; that is, there is a higher probability of recording a wind speed below the average wind speed during a sufficiently large time interval. Such distributions, which have a shape factor between $\delta=1$ and 3 are most commonly found in nature. Beyond $\delta=3$, the PDF becomes almost symmetric about the average wind speed. The scale parameter, $\beta$, on the other hand, is used to control the average value of the distribution and, hence, can be used to scale the PDF.

Using Equation (9), the statistical averages are obtained as

$$
\langle\bar{U}\rangle=\beta \Gamma\left[1+\frac{1}{\delta}\right], \quad\left\langle\bar{U}^{2}\right\rangle=\beta^{2} \Gamma\left[\frac{2+\delta}{\delta}\right],
$$

where $\Gamma[n]=(n-1)$ ! is the Gamma Function. It is worth noting that the deterministic case is recovered when $\delta \rightarrow \infty$, since $\langle\bar{U}\rangle \rightarrow \beta$ and $\left\langle\bar{U}^{2}\right\rangle \rightarrow \beta^{2}=\langle\bar{U}\rangle^{2}$.

The scale parameter, $\beta$, can be eliminated from the response statistics by expressing it in terms of the average speed of the distribution, that is $\beta=\langle\bar{U}\rangle / \Gamma\left[1+\frac{1}{\delta}\right]$. Next, we substitute Equation (10) into Equation (8) 
to obtain the following expression for the average power:

$$
\frac{\langle P\rangle}{P_{0}}=\frac{4}{3} \frac{\mu}{a_{3}\left(\zeta_{e}+\zeta_{m}\right)}\langle\bar{U}\rangle\left(\eta \frac{a_{1} \mu}{\zeta_{e}+\zeta_{m}}\langle\bar{U}\rangle-1\right)
$$

where $\eta=\frac{\Gamma\left[\frac{2+\delta}{\delta}\right]}{\Gamma\left[\frac{1+\delta}{\delta}\right]^{2}}$ is a measure of the influence of the shape of the PDF on the average output power and will be denoted in this paper as the distribution parameter. We can also define a critical cut-in average wind speed below which the harvester provides zero power for a given distribution. This speed is obtained by setting the right-hand side of Equation (11) to zero, which yields

$$
\langle\bar{U}\rangle_{c u t}=\frac{\zeta_{e}+\zeta_{m}}{\eta \mu a_{1}} .
$$

For a given average wind speed and electric load, the average output power increases with the distribution parameter $\eta$, which, in turn, as depicted in Fig. 5 (a), decreases as $\delta$ increases. In other words, for all PDFs with the same average wind speed, an exponentially correlated PDF produces higher average power levels than a Rayleigh distribution $(\delta=2)$, which, in turn, produces higher average power levels than a known wind speed located at the distribution average $(\delta \rightarrow \infty)$.

Figure 5 (b) clearly illustrates these findings for different values of $\delta$. It further illustrates that the average cut-in wind speed increases as $\delta$ increases. It is worth noting that all results presented in the paper are obtained for the galloping energy harvester whose design parameters are detailed in Appendix A2.

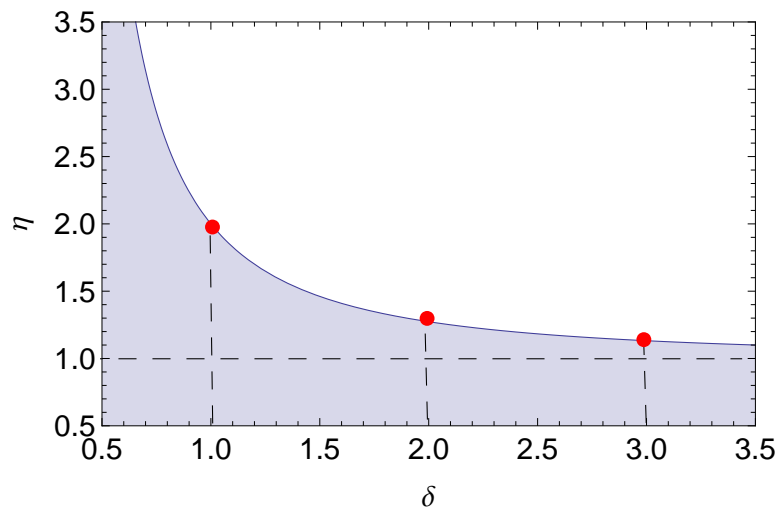

(a)

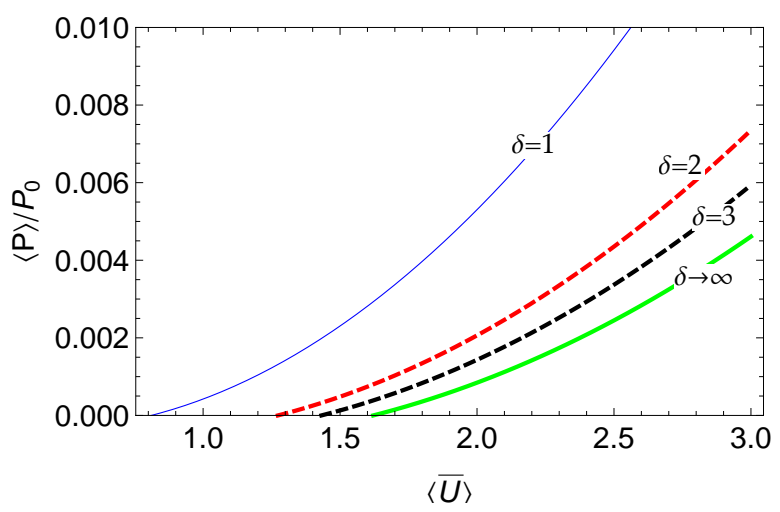

(b)

Figure 5: (a) Variation of the distribution parameter with the shape parameter. (b) Variation of the average power with the average wind speed for different values of $\delta$. Typical wind shape parameters lie within a range between 1 and 3 [15]. See Table 1 in the paper for more specific values.

\section{Optimal Load}

The electric load, embedded within the time constant ratio, $\alpha$, is a key parameter which controls the energy flow from the mechanical to the electrical subsystems. To optimize the electrical load, we solve $\partial P /\left.\partial \alpha\right|_{\alpha_{o p t}}=$ 
0 for $\alpha_{o p t}$ subject to $\partial^{2} P /\left.\partial \alpha^{2}\right|_{\alpha_{o p t}}<0$ and obtain

$$
\begin{aligned}
\alpha_{\text {opt } 1} & =1, \quad 0 \leq \zeta_{m} \leq a_{1} \eta \mu\langle\bar{U}\rangle-\frac{\kappa}{2} \\
\alpha_{\text {opt } 2,3} & =\frac{\kappa \pm \sqrt{\kappa^{2}-4\left(\eta \mu a_{1}\langle\bar{U}\rangle-\zeta_{m}\right)^{2}}}{2\left(\eta \mu a_{1}\langle\bar{U}\rangle-\zeta_{m}\right)}, \quad a_{1} \eta \mu\langle\bar{U}\rangle-\frac{\kappa}{2} \leq \zeta_{m} \leq a_{1} \eta \mu\langle\bar{U}\rangle
\end{aligned}
$$

As evident in Equation (13), there are three optimal values for the time constant ratio. The first, $\alpha_{o p t 1}=1$, which is independent of the design parameters and wind speed statistics, represents a global maximum in the average power curve when $0 \leq \zeta_{m} \leq a_{1} \eta \mu\langle\bar{U}\rangle-\frac{\kappa}{2}$. Outside this range, this global maximum becomes a local minimum, and gets replaced by two coexisting global maxima in the range $a_{1} \eta \mu\langle\bar{U}\rangle-\frac{\kappa}{2} \leq \zeta_{m} \leq a_{1} \eta \mu\langle\bar{U}\rangle$. The range in which these solutions coexist is bounded by the magnitude of the electromechanical coupling, $\kappa$, which tends to be very small. For values of $\zeta_{m}$ larger than $a_{1} \eta \mu\langle\bar{U}\rangle$, the wind loading cannot overcome the intrinsic damping and no energy can be harnessed from the flow regardless of the how the electric load is optimized. This indicates that, for small values of the damping ratio and similar to a linear system, the average power can be maximized when $\alpha_{\text {opt }}$ is set to unity. However, as the mechanical damping ratio increases, the design parameters and wind speed statistics play an important role in the optimality of the electric load.

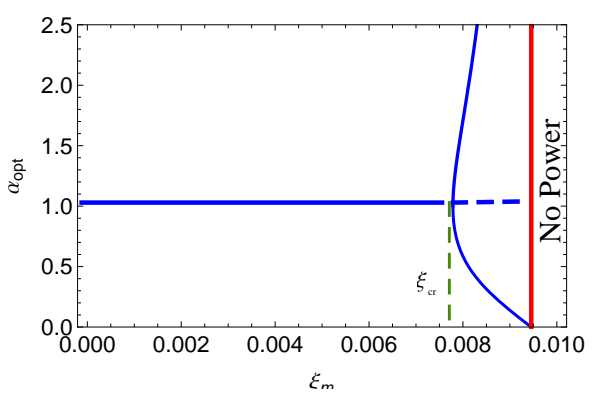

(a)

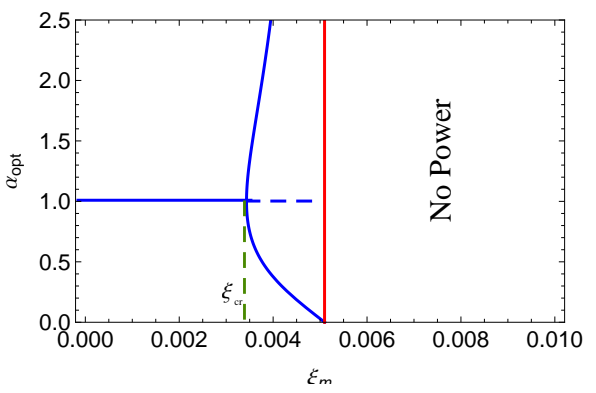

(c)

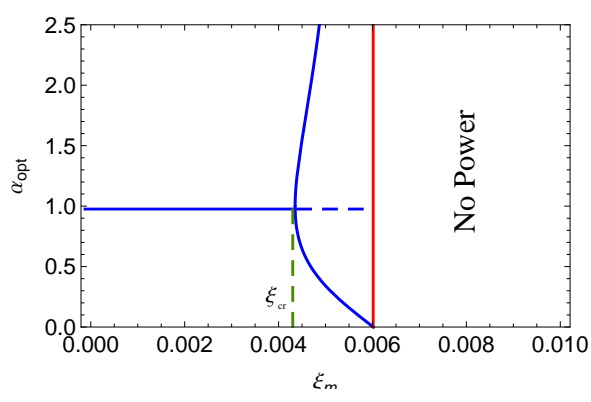

(b)

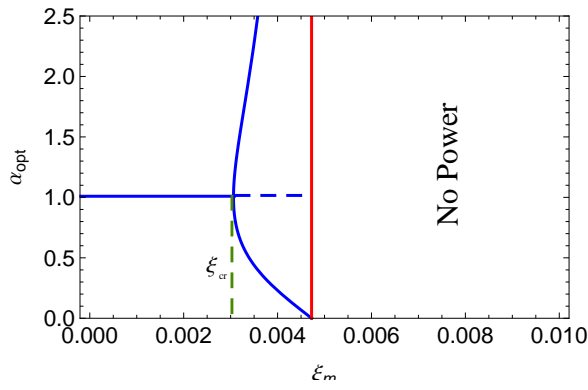

(d)

Figure 6: Variation of $\alpha_{\text {opt }}$ with the mechanical damping for different values of $\delta$. (a) $\delta=1$, (b) $\delta=2$, (c) $\delta=4$, and (d) $\delta \rightarrow \infty$.

These findings are better illustrated in Fig. 6 which depicts bifurcation diagrams of the optimal load as function of the mechanical damping ratio for different values of $\delta$. When $\delta=1$, the optimal time constant 
ratio is $\alpha=1$ up to the critical value $\zeta_{c r}=0.0077$ beyond which two global maxima corresponding to $\alpha_{o p t 2,3}$ appear. These maxima continue to coexist up to a damping ratio, $\zeta_{m}=0.0090$, beyond which no power can be harvested. As the shape parameter $\delta$ increases, $\zeta_{c r}$ decreases but the general behavior remains the same. Figure 7 depicts the average power curves for $\delta=1$ and several mechanical damping ratios further illustrating the switch from a single global maxima, corresponding to $\alpha=1$ when $\zeta_{m}=0.005$, to two global maxima when $\zeta_{m}=0.008$ and 0.0087 .

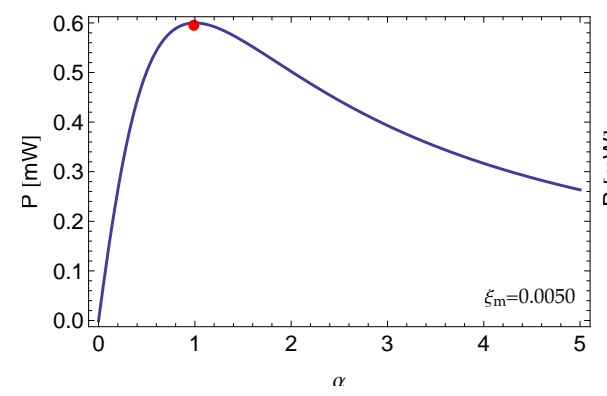

(a)

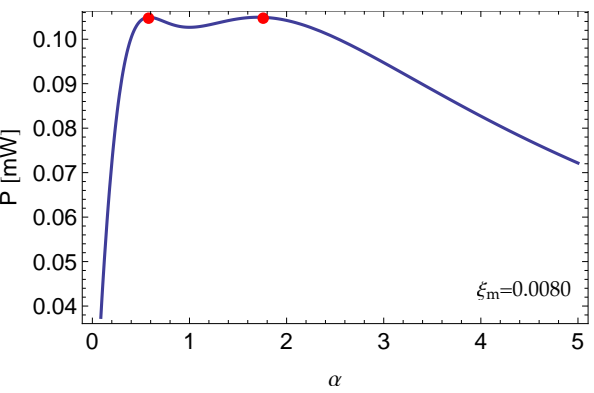

(b)

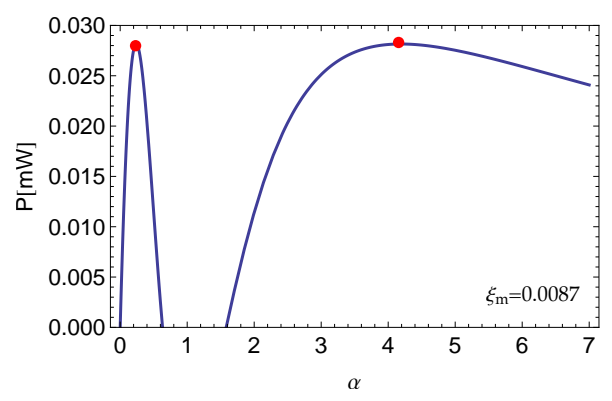

(c)

Figure 7: Variation of average power with $\alpha$ for different values of $\zeta_{m}$

\section{Case Study}

Next, we evaluate the performance of the harvester using actual wind statistics collected from different reporting stations in the UK [15]. Data histograms are first generated to show the percentage of time the wind speed was in a particular range. The corresponding Weibull PDF is then determined by finding the parameters $\delta$ and $\beta$ that best fit the reported data as shown in Fig. 8. For the chosen locations, the resulting parameters are listed in Table 1 along with the distribution parameter, $\eta$, the mean velocity $\langle\bar{U}\rangle$, the optimal load, $\alpha_{o p t}$, and the optimal average power, $\langle P\rangle$. Two values of the average power are listed, namely, that corresponding to the actual wind statistics, and that obtained by neglecting the actual wind distribution, i.e., when $\delta \rightarrow \infty$.

As listed in Table 1, it is evident that there are large discrepancies between the average optimal power levels as obtained by incorporating the actual wind statistics and those obtained assuming $\delta \rightarrow \infty$. The 
discrepancies increase as the distribution parameter $\eta$ increases or as $\delta$ decreases. Furthermore, results demonstrate that there is no direct correlation between the average wind speed at a given location and the actual average power generated. For instance, while the average winds recorded at London, Heathrow are higher than those reported in Southampton, the associated optimal average power levels are slightly higher in Southampton.

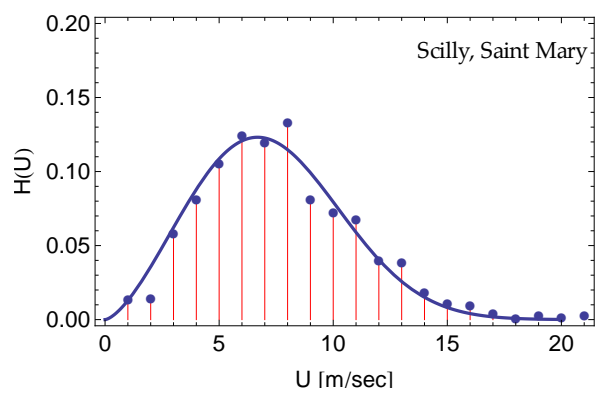

(a)

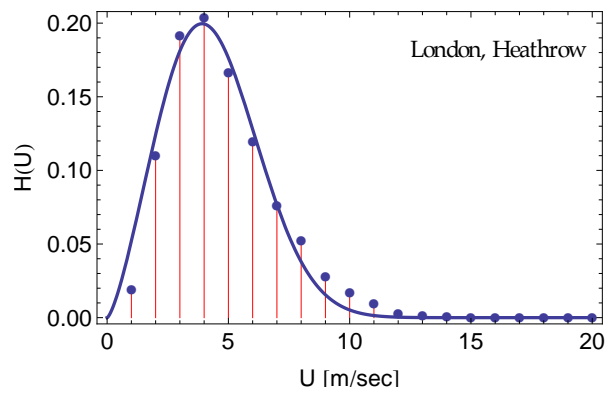

(c)

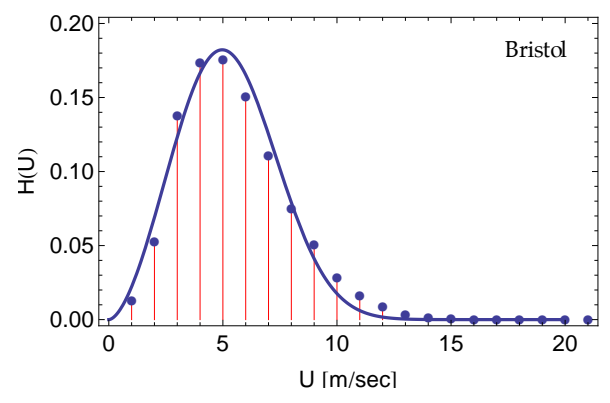

(b)

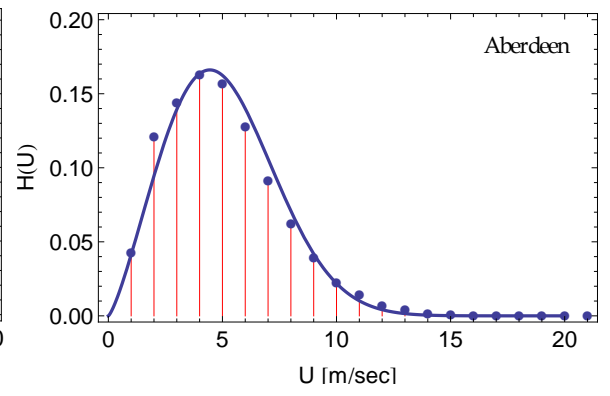

(d)

Figure 8: Wind speed histograms and associated Weibull PDFs for different locations in the UK. Data is obtained from Ref. [15].

\section{Wind Direction}

The results presented in the previous section do not incorporate the direction of the wind into the average power calculation. The underlying assumption is that all the available wind has a single direction which faces the galloping prism. However, in a realistic situation, not only does the wind speed vary with time but also its direction. As such, the wind statistics is governed by a joint $\operatorname{PDF}, F(\bar{U}, \theta)$ which involves both of the wind speed, $\bar{U}$ and its direction, $\theta$. As a result, in general, the statistical moments of the wind speed can be obtained using

$$
\left\langle\bar{U}^{n}\right\rangle=\int_{0}^{2 \pi} \int_{0}^{\infty} \bar{U}^{n} F(\bar{U}, \theta) d \bar{U} d \theta
$$

The research on modeling the joint PDF of wind speed and direction is vast with most of the research studies showing that the wind statistics can be adequately approximated by treating the wind speed and direction as 
Table 1: PDF shape/scale parameters and average output power for different locations in the UK.

\begin{tabular}{|c||c|c|c|c|c|c|c|}
\hline Reporting Station & $\beta$ & $\delta$ & $\langle\bar{U}\rangle$ & $\eta$ & $\alpha_{\text {opt }}$ & $\langle P\rangle_{\delta \rightarrow \infty}(\mathrm{mW})$ & $\langle P\rangle(\mathrm{mW})$ \\
\hline Scilly, Saint Mary & 8.2 & 2.5 & 6.44 & 1.18 & 1 & 6.10 & 7.56 \\
Bristol & 5.9 & 2.7 & 4.64 & 1.15 & 1 & 2.7 & 3.40 \\
\hline London, Heathrow & 4.9 & 2.4 & 3.84 & 1.19 & 1 & 1.66 & 2.2 \\
\hline Aberdeen & 5.7 & 2.3 & 5.05 & 1.21 & 1 & 3.38 & 4.45 \\
\hline Pembrey Sands & 6.9 & 1.60 & 5.47 & 1.41 & 1 & 4.13 & 6.53 \\
\hline Southend-On-Sea & 5.2 & 1.95 & 4.07 & 1.28 & 1 & 1.95 & 2.88 \\
\hline Southampton & 4.4 & 1.6 & 3.49 & 1.41 & 1 & 1.27 & 2.22 \\
\hline
\end{tabular}

two independent random variables [16-19]. This permits expressing the joint PDF as $F(\bar{U}, \theta)=H(\bar{U}) G(\theta)$. In such a scenario, the equation governing the statistical moments can be reduced to

$$
\left\langle\bar{U}^{n}\right\rangle=\int_{0}^{2 \pi} G(\theta) d \theta \int_{0}^{\infty} \bar{U}^{n} H(\bar{U}) d \bar{U},
$$

Using Equation (15), the conditional statistical moments of the wind spped for a given wind direction, $\theta_{e}$, can be written as

$$
\left\langle\bar{U}^{n}\left(\theta=\theta_{e}\right)\right\rangle=g\left(\theta_{e}\right) \int_{0}^{\infty} \bar{U}^{n} H(\bar{U}) d \bar{U},
$$

where $g\left(\theta_{e}\right)=\left.\int_{0}^{2 \pi} G(\theta) d \theta\right|_{\theta=\theta_{e}}$. Equation (16) is used to obtain $\langle\bar{U}\rangle$ and $\left\langle\bar{U}^{2}\right\rangle$ for a given wind direction, which can then be substituted into Equation (11) to determine the wind direction that produces maximum power levels. Alternatively, it is also plausible to generate a Weibull PDF for each direction and use it in conjunction with Equation (11) to obtain the average power.

We consider an example in which the discrete wind probability distribution for both speed and direction is known as listed in Table 2 [20]. Using the probabilities listed in Table 2, the wind rose shown in Fig. 9(a) is generated clearly illustrating that East is the direction of prevailing wind. Based on this data, it is possible to erroneously predict that facing East would be the optimal orientation of the harvester. However, when calculating the optimal power using Equation (7) and generating the power rose shown in Fig. 9(b), it becomes evident that maximum power is generated when the harvester is facing the WNW direction further illustrating the importance of considering the actual speed probability distribution when deciding the optimal orientation of the harvester.

\section{Conclusions}

Through an analytical treatment and several case studies, this paper illustrated the importance of incorporating the actual probability density function of the wind speed and direction when characterizing the performance of galloping energy harvesters. It has been shown that knowledge of the actual PDF is essential 
Table 2: Wind statistics (percentages) in terms of the speed range and direction. Data taken from [20].

\begin{tabular}{|c|c|c|c|c|c|c|c|c|c|c|c|c|c|c|c|c|}
\hline Speed Range $(\mathrm{m} / \mathrm{sec})$ & $\mathrm{N}$ & NNE & NE & ENE & E & ESE & SE & SSE & S & SSW & SW & WSW & W & WNW & NW & NNW \\
\hline $0-2$ & 0 & 0 & 0 & 0 & 0 & 0 & 0 & 0 & 0 & 0 & 0 & 0 & 0 & 0 & 0 & 0 \\
$2-4$ & 0.876 & 1.001 & 0.814 & 1.502 & 2.315 & 1.252 & 1.189 & 1.377 & 0.751 & 0.501 & 0.375 & 0.501 & 0.751 & 1.502 & 1.252 & 1.064 \\
$4-6$ & 2.378 & 3.004 & 3.191 & 5.882 & 6.821 & 4.38 & 3.88 & 4.13 & 1.877 & 0.939 & 0.563 & 0.688 & 1.377 & 2.879 & 3.379 & 2.566 \\
$6-8$ & 1.314 & 1.377 & 2.253 & 2.879 & 2.128 & 2.128 & 2.065 & 2.441 & 0.814 & 0.25 & 0.063 & 0.25 & 0.563 & 2.128 & 2.003 & 1.627 \\
$8-10$ & 0.313 & 0.313 & 0.626 & 0.751 & 0.501 & 0.501 & 0.688 & 0.751 & 0.188 & 0.063 & 0.125 & 0.063 & 0.188 & 1.064 & 1.001 & 0.438 \\
$10-12$ & 0.125 & 0.063 & 0.063 & 0.125 & 0.125 & 0.125 & 0.188 & 0.125 & 0 & 0.063 & 0 & 0 & 0.125 & 0.438 & 0.25 & 0.063 \\
$12-14$ & 0 & 0.063 & 0 & 0 & 0 & 0.063 & 0 & 0.063 & 0.125 & 0 & 0 & 0.063 & 0.063 & 0.125 & 0.063 & 0.063 \\
$14-16$ & 0 & 0.063 & 0 & 0 & 0 & 0.063 & 0.063 & 0.063 & 0 & 0 & 0 & 0 & 0 & 0 & 0.063 & 0 \\
$16-18$ & 0 & 0 & 0 & 0 & 0 & 0 & 0 & 0 & 0 & 0 & 0 & 0 & 0 & 0.063 & 0 & 0 \\
$18-20$ & 0 & 0 & 0.125 & 0 & 0 & 0 & 0 & 0 & 0 & 0 & 0 & 0 & 0 & 0 & 0 & 0 \\
$20-22$ & 0 & 0 & 0 & 0 & 0 & 0 & 0 & 0 & 0 & 0 & 0 & 0 & 0 & 0 & 0 & 0 \\
$22-24$ & 0 & 0 & 0 & 0.063 & 0 & 0 & 0 & 0 & 0 & 0 & 0 & 0 & 0 & 0 & 0 & 0 \\
$24-26$ & 0 & 0 & 0 & 0 & 0 & 0 & 0 & 0 & 0 & 0 & 0 & 0 & 0 & 0 & 0 & 0 \\
$26-28$ & 0 & 0 & 0 & 0 & 0.063 & 0 & 0 & 0 & 0 & 0 & 0 & 0 & 0 & 0 & 0 & 0 \\
\hline
\end{tabular}

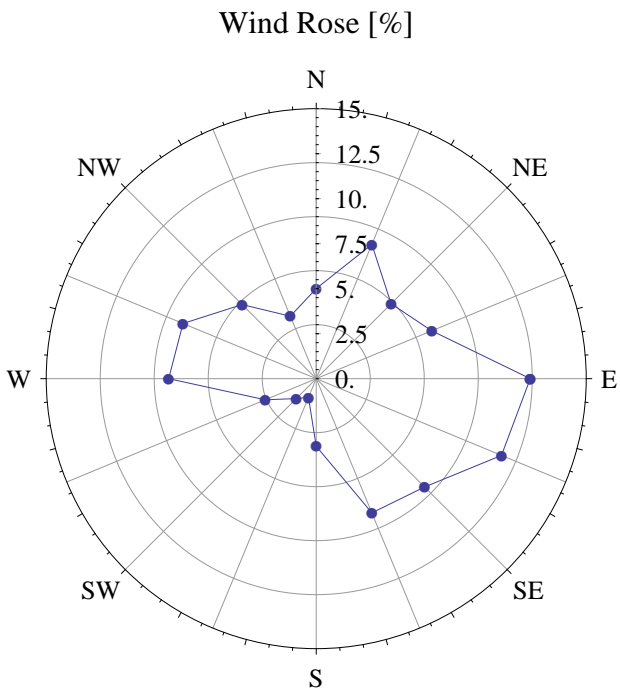

(a)

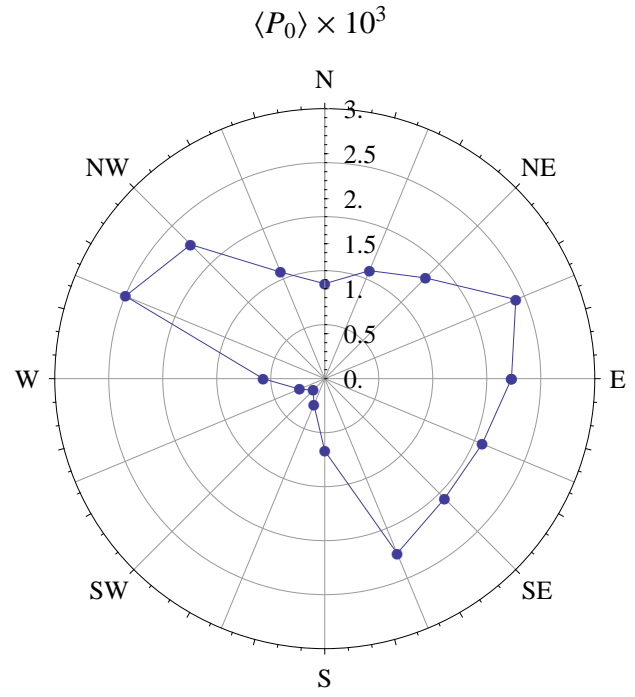

(b)

Figure 9: (a) Wind rose illustrating wind percentages in any given direction. (b) Power rose illustrating the average power in any given direction.

for correct power predictions as well as for accurate electric load optimization. Furthermore, by incorporating the wind statistics into the analysis, it was demonstrated that the direction of the prevailing wind may not necessarily be the ideal direction to maximize the average power. Such knowledge is essential prior to designing small galloping energy harvesters, because deviating from the optimal operating conditions can drop their small energy outputs even further making the energy harvesting process inefficient.

To arrive at the previous general conclusions, the paper presented a deterministic model of a piezoelectric galloping energy harvester. An approximate analytical solution of the model is then presented using the method of multiple scales. The resulting solution is used to obtain an expression for the average output 
power of the harvester as function of the wind statistical averages, which, in turn, were obtained by fitting the wind probability density function into a Weibull distribution. The following specific conclusions were also deduced:

1. In addition to the design parameters of the harvester, the average output power depends on a single parameter called the distribution parameter. This parameter captures the influence of both of the shape and scale parameters of the Weibull distribution on the average power.

2. The average output power increases as the distribution parameter is increased. In other words, for all PDFs having the same average wind speed, an exponentially correlated PDF produces higher average power levels than a Rayleigh distribution, which, in turn, produces higher average power levels than a known wind speed located at the distribution average.

3. Upon optimizing the average output power with respect to the electric load resistance, three different optimal electric loads are obtained. For small values of the mechanical damping ratio, the average power can be maximized by setting the time constant ratio (ratio between the time constant of the harvesting circuit and the period of the mechanical oscillator) to unity regardless of the design or statistical parameters. However, as the mechanical damping ratio increases beyond a critical value, the design parameters and wind speed statistics play an important role in the optimality of the electric load.

It is worth mentioning that similar analyses involving wind speed statistics are regularly implemented to determine the optimal design parameters of horizontal-axis wind turbines with key differences appearing in the models and expressions used for the average output power.

\section{Appendices}

\section{A.1 Appendix A1: Approximate Solution of the Equations of Motion}

We utilize the method of multiple scales [21] to obtain an approximate analytical solution of Equations (3). To that end, we expand the time dependence in the equation into multiple time scales in the form

$$
T_{n}=\varepsilon^{n} t, \quad n=0,1, \ldots
$$

where $\varepsilon$ is a bookkeeping parameter. The time derivatives can be further expressed as

$$
\begin{aligned}
\frac{d}{d t} & =D_{0}+\varepsilon D_{1}+O\left(\varepsilon^{2}\right), \\
\frac{d^{2}}{d t^{2}} & =D_{0}^{2}+2 \varepsilon D_{0} D_{1}+O\left(\varepsilon^{2}\right),
\end{aligned}
$$

where $D_{n}=\frac{\partial}{\partial T_{n}}$. Furthermore, we expand $y$ and $v$ in the following form:

$$
\begin{aligned}
& y(t)=y_{0}\left(T_{0}, T_{1}\right)+\varepsilon y_{1}\left(T_{0}, T_{1}\right)+O\left(\varepsilon^{2}\right), \\
& v(t)=v_{0}\left(T_{0}, T_{1}\right)+\varepsilon v_{1}\left(T_{0}, T_{1}\right)+O\left(\varepsilon^{2}\right) .
\end{aligned}
$$


The coefficients in Equation (3) are scaled such that the effect of mechanical damping appears at the first order of the perturbation problem, $O(\varepsilon)$, as well as the aerodynamic forcing and backward coupling. In other words, we let

$$
\zeta_{m}=\varepsilon \zeta_{m}, \quad a_{1}=\varepsilon a_{1}, \quad a_{3}=\varepsilon a_{3}, \quad \kappa=\varepsilon \kappa .
$$

Substituting Equations (A.2)-(A.4) back into Equations (3), then collecting terms of equal powers of $\varepsilon$ yields $O\left(\epsilon^{0}\right):$

$$
\begin{gathered}
D_{0}^{2} y_{0}+y_{0}=0, \\
D_{0} v_{0}+\alpha v_{0}=-D_{0} y_{0},
\end{gathered}
$$

$O(\epsilon)$ :

$$
\begin{gathered}
D_{0}^{2} y_{1}+y_{1}=-2 D_{0} D_{1} y_{0}-\left(2 \zeta_{m}-\mu a_{1} \bar{U}\right) D_{0} y_{0}+\kappa v_{0}-\frac{2 \mu a_{3}}{\bar{U}} y_{0}^{3} \\
D_{0} v_{1}+\alpha v_{1}=-\left(D_{0} y_{1}+D_{1} y_{0}\right)-D_{1} v_{0}
\end{gathered}
$$

The solution of the zeroth order perturbation equations, Equations (A.5a) and (A.5b), can be written as:

$$
\begin{gathered}
y_{0}=A\left(T_{1}\right) e^{i T_{0}}+c c, \\
v_{0}=Z A\left(T_{1}\right) e^{i T_{0}}+c c,
\end{gathered}
$$

where $c c$ is the complex conjugate, $A\left(T_{1}\right)$ is a complex valued function to be determined at a later stage of the analysis, and $Z=\frac{i}{i+\alpha}$. Substituting Equations (A.7a) and (A.7b) into Equation (A.6a), and eliminating the secular terms (terms that have the coefficient $e^{ \pm i T_{0}}$ ), yields

$$
-2\left(\zeta_{m}-\mu a_{1} \bar{U}\right) i A-2 i D_{1} A-i \frac{\kappa}{i+\alpha} A+\frac{6 \mu a_{3}}{\bar{U}} A^{2} \bar{A}=0,
$$

where $\bar{A}$ is the complex conjugate of $A$. To solve Equation (A.8), we find it convenient to introduce the polar transformation $A\left(T_{1}\right)=1 / 2 a\left(T_{1}\right) e^{i \gamma\left(T_{1}\right)}$, then separate the real and imaginary parts from the outcome to obtain

$$
\begin{gathered}
D_{1} a=-\left(\zeta_{m}+\zeta_{e}-\mu a_{1} \bar{U}\right) a-\frac{3}{4} \frac{\mu a_{3}}{\bar{U}} a^{3}, \\
D_{1} \gamma=\frac{\kappa}{2} \frac{\alpha}{\alpha^{2}+1}, \quad a \neq 0 .
\end{gathered}
$$

Note that the amplitude dynamics is completely decoupled from that associated with the phase, $\gamma$. As such, the steady-state response amplitude can be obtained by setting the time derivative in Equation (A.9a) to zero and obtaining:

$$
\begin{aligned}
a_{10} & =0 \\
a_{20,30} & = \pm \frac{2}{\sqrt{3}} \frac{\left(\zeta_{m}+\zeta_{e}\right)}{\mu} \sqrt{\frac{\mu}{\left(\zeta_{e}+\zeta_{m}\right) a_{3}}\left(\frac{a_{1} \mu}{\left(\zeta_{e}+\zeta_{m}\right)} \bar{U}-1\right)} \bar{U}, \quad \frac{a_{1} \mu}{\left(\zeta_{e}+\zeta_{m}\right)} \bar{U}-1>0 .
\end{aligned}
$$


The stability of the steady-state solutions is assessed by evaluating the Jacobian of Equation (A.9a) at the roots. This yields the following stable regions:

$$
\begin{aligned}
a_{10} & =0, \quad \text { when } \frac{a_{1} \mu}{\left(\zeta_{e}+\zeta_{m}\right)} \bar{U}-1 \leq 0, \\
a_{20,30} & = \pm \frac{2}{\sqrt{3}} \frac{\left(\zeta_{m}+\zeta_{e}\right)}{\mu} \sqrt{\frac{\mu}{\left(\zeta_{e}+\zeta_{m}\right) a_{3}}\left(\frac{a_{1} \mu}{\left(\zeta_{e}+\zeta_{m}\right)} \bar{U}-1\right)} \bar{U}, \quad \text { when } \quad \frac{a_{1} \mu}{\left(\zeta_{e}+\zeta_{m}\right)} \bar{U}-1>0 .
\end{aligned}
$$

Based on the stability analysis of the roots, one can correctly surmise that $\bar{U}=\frac{\zeta_{e}+\zeta_{m}}{a_{1} \mu}$ represents a supercritical picthfork bifurcation in the amplitude dynamics, or a Hopf bifurcation in the original dynamics. The steady-state phase can be written as

$$
\gamma=\epsilon \frac{\kappa}{2} \frac{\alpha}{\alpha^{2}+1} T_{0}+\gamma_{0}
$$

Substituting Equation (A.12) and (A.10) into Equation (A.7b), we obtain the following approximate steadystate analytical solution for the non-trivial responses:

$$
\begin{array}{r}
y=\frac{2}{\sqrt{3}} \frac{\left(\zeta_{m}+\zeta_{e}\right)}{\mu} \sqrt{\frac{\mu}{\left(\zeta_{e}+\zeta_{m}\right) a_{3}}\left(\frac{a_{1} \mu}{\left(\zeta_{e}+\zeta_{m}\right)} \bar{U}-1\right)} \bar{U} \cos \left(\left(1+\epsilon \frac{\kappa}{2} \frac{\alpha}{\alpha^{2}+1}\right) t+\gamma_{0}\right) \\
v=\frac{2}{\sqrt{3}} \frac{\left(\zeta_{m}+\zeta_{e}\right)}{\mu \sqrt{1+\alpha^{2}}} \sqrt{\frac{\mu}{\left(\zeta_{e}+\zeta_{m}\right) a_{3}}\left(\frac{a_{1} \mu}{\left(\zeta_{e}+\zeta_{m}\right)} \bar{U}-1\right) \bar{U}} \cos \left(\left(1+\epsilon \frac{\kappa}{2} \frac{\alpha}{\alpha^{2}+1}\right) t+\gamma_{0}-\arctan \alpha\right) \cdot(\mathrm{A}
\end{array}
$$

\section{A.2 Appendix A2: Numerical Parameters}

The galloping energy harvester used to generate the simulations presented in this paper is shown in Fig. 10. The harvester was modeled and its response was validated experimentally by the author and his colleagues in Ref. [22]. The harvester consists of a stainless-steel cantilever beam fixed at one end and free to oscillate at the other. A square-sectioned bluff body with dimensions of $50 \times 50 \times 100 \mathrm{~mm}^{3}$ is attached to the free end and is placed normal to the direction of the flow. The stainless beam has dimensions of $209 \times 24 \times$ $1 \mathrm{~mm}^{3}$, while the piezoelectric layer attached to the beam has dimensions of $95 \times 28 \times 0.2 \mathrm{~mm}^{3}$. The experimentally-identified geometric and material properties of the system are listed in Table 3.

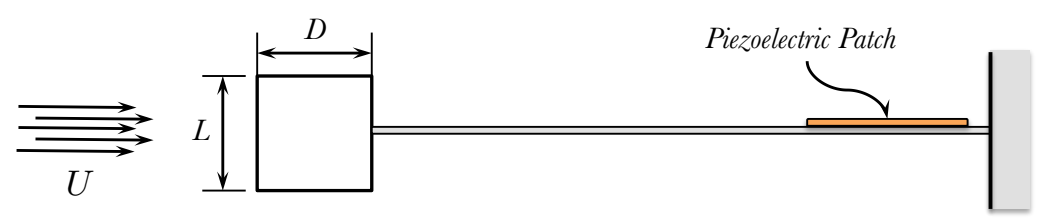

Figure 10: A schematic of the galloping energy harvester used by Bibo et al. [22] 
Table 3: Material and geometric properties of the harvester.

\begin{tabular}{cl}
\hline Parameter (symbol) & Value \\
\hline Cantilever beam length & $0.209 \mathrm{~m}$ \\
Effective mass $(M)$ & $0.1134 \mathrm{Kg}$ \\
Damping ratio $\left(\zeta_{m}\right)$ & 0.003 \\
Air Density $(\rho)$ & $1.25 \mathrm{Kg} / \mathrm{m}^{3}$ \\
Capacitance of piezoelectric layer $\left(C_{p}\right)$ & $187 \mathrm{nF}$ \\
Electromechanical coupling $(\theta)$ & $1.9 \times 10^{-4} \mathrm{~N} / \mathrm{V}$ \\
Prism height $(L)$ & $0.1 \mathrm{~m}$ \\
Cross flow dimension $(D)$ & $0.05 \mathrm{~m}$ \\
Linear aerodynamic coefficient $\left(a_{1}\right)$ & 2.5 \\
Cubic aerodynamic coefficient $\left(a_{3}\right)$ & 130 \\
\hline
\end{tabular}

\section{Acknowledgment}

This material is based upon work supported by the National Science Foundation under CAREER Grant No. 1055419. Any opinions, findings, and conclusions or recommendations expressed in this material are those of the author and do not necessarily reflect the views of the National Science Foundation.

\section{References}

[1] HD. Akaydın, N. Elvin, and Y. Andreopoulos. Wake of a Cylinder: A Paradigm for Energy Harvesting with Piezoelectric Materials. Experiments in Fluids, 49(1):291-304, 2010.

[2] H. Jung and S. Lee. The Experimental Validation of a New Energy Harvesting System Based on the Wake Galloping Phenomenon. Smart Materials and Structures, 20(5):055022, 2011.

[3] A. Erturk, W. Vieira, C. De Marqui Jr, and D. Inman. On the Energy Harvesting Potential of Piezoaeroelastic Systems. Applied Physics Letters, 96(18):184103, 2010.

[4] M. Bryant and E. Garcia. Modeling and Testing of a Novel Aeroelastic Flutter Energy Harvester. Journal of Vibration and Acoustics, 133(1):011010, 2011.

[5] VC. Sousa, M. de M Anicézio, C. De Marqui Jr, and A. Erturk. Enhanced Aeroelastic Energy Harvesting by Exploiting Combined Nonlinearities: Theory and Experiment. Smart Materials and Structures, 20(9):094007, 2011. 
[6] J. Sirohi and R. Mahadik. Harvesting Wind Energy Using a Galloping Piezoelectric Beam. Journal of Vibration and Acoustics, 134(1):011009, 2012.

[7] J. Sirohi and R. Mahadik. Piezoelectric Wind Energy Harvester for Low-power Sensors. Journal of Intelligent Material Systems and Structures, page 1045389X11428366, 2011.

[8] A. Abdelkefi, M. Hajj, and A .H. Nayfeh. Piezoelectric Energy Harvesting from Transverse Galloping of Bluff Bodies. Smart Materials and Structures, 22(1):015014, 2013.

[9] Y. Yang, L. Zhao, and L. Tang. Comparative Study of Tip Cross-sections for Efficient Galloping Energy Harvesting. Applied Physics Letters, 102(6):064105, 2013.

[10] JM. Kluger, FC. Moon, and RH. Rand. Shape Optimization of a Blunt Body Vibro-wind Galloping Oscillator. Journal of Fluids and Structures, 40:185-200, 2013.

[11] A. Bibo and M. F. Daqaq. On the Optimal Performance and Universal Design Curves of Galloping Energy Harvesters. Applied Physics Letters, 104(2):023901, 2014.

[12] M. Paidoussis, S. Price, and E. deLangre. Fluid Structural Interactions: Cross-Flow Induced Instabilities. Cambridge University Press, 2011.

[13] GV. Parkinson and JD. Smith. The Square Prism as an Aaeroelastic Non-linear Oscillator. The Quarterly Journal of Mechanics and Applied Mathematics, 17(2):225-239, 1964.

[14] G. M. Masters. Renewable and Efficient Power Systems. John Wiley \& Sons, 2004.

[15] http://www.rensmart.com/weather/windarchive.

[16] B. McWilliams, MM. Newmann, and D. Sprevak. The Probability Distribution of Wind Velocity and Direction. Wind Engineering, 3(296):73, 1979.

[17] B. McWilliams and D. Sprevak. The Estimation of the Parameters of the Distribution of Wind Speed and Direction. Wind Engineering, 4(227):15, 1980.

[18] RO. Weber. Estimators for the Standard Deviation of Horizontal Wind Direction. Journal of Appl Meteorology, 28(213):24, 1997.

[19] R. Garcia-Rojo. Algorithm for the Estimation of the Long-term Wind Climate at a Meteorological Mast Using a Joint Probabilistic Approach. Journal of Applied Meteorology, 28(213):24, 2004.

[20] J. Chen and X. Zhang. An emprirical joint probability desnity function of wind speed and direction. In In Proceedings of The Seventh Asia-Pacific Conference on Wind Engineering.

[21] A. H. Nayfeh. Introduction to Perturbation Techniques. John Wiley \& Sons, 2011.

[22] A. Bibo, A. Alhadidi, and M.F. Daqaq. Exploiting a Nonlinear Restoring Force to Improve the Bandwidth of Galloping Energy Harvesters. Journal of Applied Physics, 117(4). 